\title{
Analysis of the Development of Avascular Necrosis in Slipped Capital Femoral Epiphysis
}

\author{
Ryan du Sart, Ruvini Thashila Gunawardana, Colin Whitewood \\ Princess Margaret Hospital, Perth, Australia \\ Email: rcdusart@yahoo.com
}

Received 9 June 2014; revised 27 July 2014; accepted 13 August 2014

Copyright (C) 2014 by authors and Scientific Research Publishing Inc.

This work is licensed under the Creative Commons Attribution International License (CC BY). http://creativecommons.org/licenses/by/4.0/

c) (i) Open Access

\begin{abstract}
Introduction: Slipped capital femoral epiphysis (SCFE) is a common hip disorder in adolescents. The most serious complication is avascular necrosis (AVN), which has lasting sequelae. The objective of this study was to determine the rate of AVN in stable slips. Method: We reviewed retrospectively all the children treated for SCFE between 2004 and 2008 at Princess Margaret Hospital. Results: Of a total of 67 patients, $51(76 \%)$ were stable slips and $16(24 \%)$ unstable. Avascular necrosis developed in 3 patients with stable slip (5.9\%) and in 6 with unstable slip (37.5\%). There was no statistically significant association found between AVN and age, weight, slip severity, pin position, number of pins used, experience of the surgeon or side affected. Those with stable presentations can develop $\mathrm{AVN}^{2}$, males are more likely and the possibility of developing $\mathrm{AVN}$ increases as the time from presentation to operation increased. Conclusion: AVN can occur in stable slips without joint penetration. This combined with male sex and increased time to operation, increases the risk of AVN.
\end{abstract}

\section{Keywords}

Avascular Necrosis, Slipped Capital Femoral Epiphysis, Stable Slips

\section{Introduction}

Slipped capital femoral epiphysis is a serious hip disorder. The aetiology of SCFE includes both biomechanical and biochemical factors that combine to create a weakened physis, with subsequent failure [1]. Mechanical factors include obesity, femoral retroversion, and increased physeal obliquity [1].

SCFE develops during puberty, a time of many hormonal changes. Rapid longitudinal growth occurs in re- 
sponse to increased levels of growth hormone. This rapid growth is associated with an increased chondrocyte proliferation rate and increased height of the zone of hypertrophy [2]. This zone is wider than normal, with disordered chondrocyte columns, decreased number of cells, smaller cells and increased number of dead and degenerative cells [3] [4].

It has many classifications but the best predictor of avascular necrosis is physeal stability described by Loder et al. [5]. Using Loder's classification we now know there is a difference between the rates of AVN in stable versus unstable hips. A slip is stable when walking and weight bearing are still possible with or without crutches, while an unstable slip is when one cannot weight bear on the limb even with cructhes.

Our objective was to determine if the rate of AVN for stable slips is $0 \%$ as proposed by Loder [5] and other authors [1] [6] [7], and the factors that influence the development of AVN in SCFE.

\section{Method}

We retrospectively reviewed medical files of all patients treated for slips at our institution between 2004 and 2008. Medical files were reviewed to determine age, sex, weight, side of slip, time from presentation to operation, any underlying endocrine problem, experience of surgeon, and whether manipulation was performed.

Using our radiological archive we reviewed their radiographs, and determined the degree of slip, number of pins used, pin position, and whether avascular necrosis developed.

The degree of displacement of the femoral epiphysis was determined from anterior-posterior and lateral films using the head-shaft angle of Southwick [8] (Figure 1). The degree of slip was calculated by subtracting the value on the normal side from the value of affected side or, in the case of simultaneous bilateral SCFE, a standard norm of 11 was used [9]. The severity of the SCFE was determined using the Boyer [10] method; "mild" SCFE had a difference in the angle on the lateral radiograph of $<30^{\circ}$, "moderate" between $30^{\circ}$ and $60^{\circ}$, and "severe" a difference $>60^{\circ}$.

The position of the screw was measured by the method developed by Mulholland and Gunn [11] and recently applied to SUFE screw position by Aronson and Carlson [12] (Figure 2). The screw was in Position 1 when its central axis was over the centre line of the femoral head or within a distance equal to one-half the diameter of the screw. The screw was in Position 2 when the distance between its axis and the centre line of the femoral head was between one-half and one screw-diameter, and the screw was in Position 3 when its axis was more than one screw-diameter from the centre line of the femoral head. The position is given as two numbers: the first

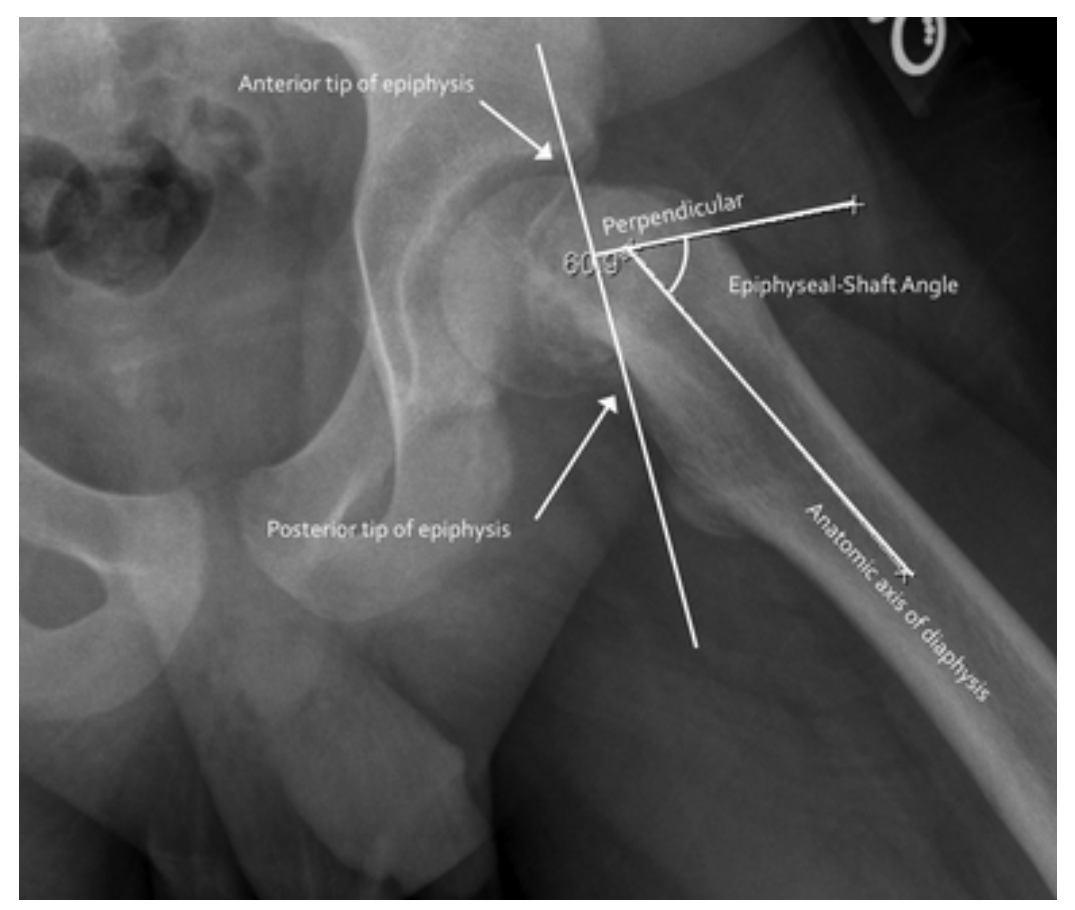

Figure 1. Head shaft angle of Southwick. 


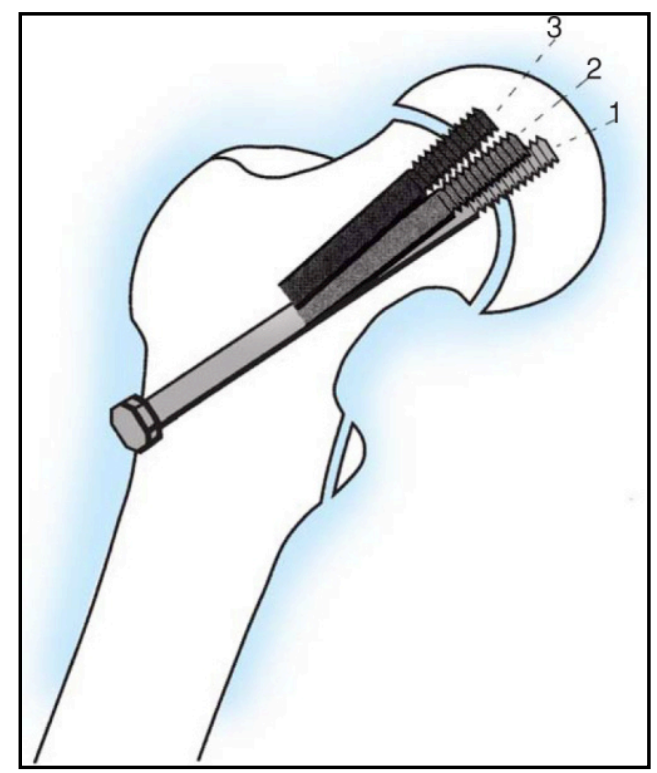

Figure 2. Screw placement.

is for the position of the screw as seen on the AP radiograph and the second is for the position as seen on the lateral radiograph.

Osteonecrosis was determined by serial radiographs showing the presence of increased density in the femoral head with segmental collapse.

Data were stored and analyzed. Logistic regression was used to determine which variables were related to the development of AVN. Simple logistic regression was used modeling AVN by each variable one at a time. Multiple logistic regression was then used to determine if these variables were related to the development of AVN in combination. Backward stepwise logistic regression which involved fitting a model with all of the variables and then working backwards to find the best-fit model.

All patients were taken to theatre, and had a general anesthetic. They were placed supine on a radiolucent table. Under sterile conditions, guide pins were placed using an image intensifier. Depending on the surgeon's preference, 1 or 2 cannulated screws $(7.3 \mathrm{~mm})$ were used as per the guidelines at our institution.

\section{Results}

Of a total of 67 patients, $51(76 \%)$ were stable slips and 16 (24\%) unstable. Avascular necrosis developed in 3 patients with stable slip (5.9\%) and in 6 with unstable slip (37.5\%). Of those that developed AVN, 8 were male and 1 female (Table 1). None of the patients that developed AVN had any endocrine problems, pin penetration of the joint surface, or any manipulation performed in theatre.

There was no statistically significant association found between AVN and age, weight, slip severity, pin position, number of pins used, experience of the surgeon or side affected (Table 2).

However, this analysis showed that the combination of stability, sex and the time from presentation to operation were all related to the development of AVN with p-values of $0.0018,0.0046$ and 0.0088 respectively. In this group of patients, those with stable slips are less likely to develop AVN, males are more likely and the likelihood of developing AVN increased as the time from presentation to operation increased (mean for AVN group was 24.1 hours and 20.4 hours for those without AVN).

The data was also examined to determine if there was a different relationship between the development of AVN and the other variables in the stable and unstable groups. None of the variables showed a significant interaction at the $5 \%$ level, however the interaction between stability and the mean time from presentation to operation approached significance at the $10 \%$ level $(\mathrm{p}$-value $=0.0766)$. Table 3 shows the mean time for the different groups with and without AVN. This shows that the stable patients who developed AVN had a very high mean time from presentation at our hospital to operation. There are only 3 such patients in this analysis and so this might warrant further investigation. 
Table 1. Stable versus unstable.

\begin{tabular}{ccc}
\hline & Stable & Unstable \\
\hline Number of patients & $51(76 \%)$ & $16(24 \%)$ \\
Sex-male & 28 & 9 \\
Sex-female & 23 & 7 \\
Age & 11.6 & 12.25 \\
AVN & $3(5.9 \%)$ & $6(37.5 \%)$ \\
\hline
\end{tabular}

Table 2. Association between AVN and different variables.

\begin{tabular}{cc}
\hline Variable & p-Value \\
Stable & $0.004^{*}$ \\
Age & 0.206 \\
Sex & 0.057 \\
Follow-up & 0.280 \\
Consultant versus registrar & 0.994 \\
Time to present for operation & 0.335 \\
Severity of slip & 0.054 \\
Number of screws & 0.077 \\
Side & 0.994 \\
Weight & 0.262 \\
Screw placement & 0.995 \\
Prophylactic pinning & 0.487 \\
\hline
\end{tabular}

\section{Table 3. Mean time from presentation to operation.}

\begin{tabular}{ccc}
\hline Mean time from presentation to operation (hours) & Stable & Unstable \\
\hline AVN & 39.0 & 16.6 \\
No AVN & 21.0 & 17.3 \\
\hline
\end{tabular}

\section{Discussion}

Avascular necrosis is a known complication of unstable slips [5] [7] [13]-[15]. When it occurs it is devastating and disconcerting to all parties. There are numerous classifications of SCFE but none have been better in predicting avascular necrosis as Loder's [5].

Loder [5] reported no AVN in 25 stable slips, and a rate of $47 \%$ in the unstable group. Tokmakova [14] also reported no AVN in the 204 treated stable slips (Table 4).

In a retrospective study done by Kenndey [7], they found that AVN did not occur in any of the stable slips $(0 / 272)$ and occurred in 4 of the unstable slips (4/27). In the unstable group, Kennedy found that a younger age at presentation was a predictor of a poorer outcome.

Apart from physeal stability, there have been numerous associations with regards to the development of AVN. Potential mechanisms for the development of osteonecrosis may be partially dependent on the following: type of treatment, kinking of periosteal blood vessels, synovitis associated with the trauma, vascular tamponade resulting from intra-capsular hematoma, timing of treatment, and location of fixation devices within the epiphysis.

Certain authors have suggested that timing of surgery may be a significant factor. However, there is no clear consensus, as earlier studies showed a higher incidence of osteonecrosis in hips which were reduced early [5] [16], compared to more recent studies, which suggest that early treatment may be beneficial. Kalogrianitis et al. 
Table 4. Rate of avascular necrosis (AVN) in the literature.

\begin{tabular}{ccc}
\hline Study & Rate of AVN (stable slips) & Rate of AVN (unstable slips) \\
\hline Our study & $5.9 \%$ & $37.5 \%$ \\
Loder [5] & 0 & $47 \%$ \\
Kennedy [7] & 0 & 14.8 \\
Tokmakova [14] & 0 & - \\
Fallath [13] & $1.4 \%$ & $21.4 \%$ \\
\hline
\end{tabular}

[17] reported on a series of 16 unstable slips, of which $50 \%$ went on to AVN, and suggested that there may be an "unsafe window" between 24 hours and 8 days. As a result, they advocated early treatment if possible or postponing treatment over a week in cases of delayed presentations. Gordon et al. [18] also reported on a series of 16 consecutive unstable slips. One of 5 patients treated more than 24 hours after the onset of acute symptoms developed osteonecrosis compared with 1 of 11 patients treated within 24 hours. The authors therefore recommended urgent reduction and pinning of the hip within 24 hours of acute symptoms. In a series of 91 acute and unstable slipped capital femoral epiphysis, 56 of which were treated with some form of internal fixation, Peterson et al. [19] reported a 7\% rate of AVN in patients treated less than 24 hours after presentation. The risk of osteonecrosis increased to $20 \%$ for those patients treated after 24 hours.

Manipulative reduction and slip severity have also been considered as risk factors for osteonecrosis [16] [20]. Tokmakova et al. [20] concluded that osteonecrosis was more likely to occur in association with worsening degrees of unstable slip, and that complete or partial reduction of an unstable SCFE increases the chance that osteonecrosis will develop. Casey et al. [16] showed increased rates of osteonecrosis in unstable slips, especially in those that were "over-reduced". Other reports have suggested that spontaneous, incidental reduction of the slip from simple positioning on the operating table does not seem to increase the risk of AVN [6] [19] [21].

Herrera [22] demonstrated increased intracapsular pressures in children with unstable SCFE and have advocated early fixation with capsular decompression to eliminate the tamponading effect of the joint effusion. Other studies have also supported the use of an arthrotomy to decompress the hematoma [18] [19]. However, in the study done by Sankar [23], they did not demonstrate a significant difference in the rate of AVN between those who did or did not undergo a capsular decompression.

Sanker [23] did however demonstrate that younger age and a shorter duration of prodromal symptoms were significant risk factors for osteonecrosis. The latter they suggested was probably due to greater degree of physeal instability in this group.

In all the literature, the rate of AVN in stable slips was $0 \%$. The only reported case of AVN in a stable slip was by Fallath [13] (1.4\%). This case required repining due to joint penetration. Placement of pins in the anterolateral portion of the epiphysis has been reported to increase the risk of AVN [24] [25], and the authors suggested that there 1 case was probably induced by pin penetration. In a search of the literature, we have only have been able to find 1 reported case of AVN in a stable slip.

Although the number of patients in our retrospective study was small (67), we found no correlation between AVN and age, weight, slip severity, pin position, number of pins used, experience of the surgeon or side affected. We however did find that stable presentations are less likely to develop AVN, males are more likely, and the likelihood of developing AVN increased as the time from presentation to operation increased.

As such, the pathomechanism of osteonecrosis seems elusive and, likely, multifactorial.

\section{Conclusion}

Our study shows that AVN can occur in stable slips, and this combined with male sex and increased time to operation, increases the risk of AVN. The authors recognize that this study had only a small sample size and study of a larger group is necessary to confirm the results.

\section{References}

[1] Aronsson, D.D., Loder, R.T., Breur, G.J. and Weinstein, S.L. (2006) Slipped Capital Femoral Epiphysis: Current Concepts. Journal of the American Academy of Orthopaedic Surgeons, 14, 666-679.

[2] Loder, R.T., Aronsson, D.D., Dobbs, M.B. and Weinstein, S.L. (2001) Slipped Capital Femoral Epiphysis. Instructional Course lectures, 50, 555-570. 
[3] Howorth, M. (1949) Slipping of the Upper Femoral Epiphysis. Journal of Bone and Joint Surgery (American Volume), 31A, 734-747.

[4] Michelson, M.R., Ponseti, I.V., Cooper, R.R. and Maynard, J.J. (1977) The Ultrastructure of the Growth Plate in Slipped Capital Femoral Epiphysis. Journal of Bone and Joint Surgery (American Volume), 59A, 1076-1081.

[5] Loder, R.T., Richards, B.S., Shapiro, P.S., Reznick, L.R. and Aronson, D.D. (1993) Acute Slipped Capital Femoral Epiphysis: The Importance of Physeal Instability. Journal of Bone and Joint Surgery (American Volume), 75, 11341140 .

[6] Kallio, P.E., Mah, E.T., Foster, B.K., Paterson, D.C. and LeQuesne, G.W. (1995) Slipped Capital Femoral Epiphysis: Incidence and Clinical Assessment of Physeal Instability. Journal of Bone and Joint Surgery (British Volume), 77, 752-755.

[7] Kennedy, J.G., Hresko, M.T., Kasser, J.R., Shrock, K.B., Zurakowski, D., Waters, P.M. and Millis, M.B. (2001) Osteonecrosis of the Femoral Head Associated with Slipped Capital Femoral Epiphysis. Journal of Pediatric Orthopaedics, 21, 189-193. http://dx.doi.org/10.1097/01241398-200103000-00011

[8] Southwick, W.O. (1967) Osteotomy through the Lesser Trochanter for Slipped Capital Femoral Epiphysis. Journal of Bone and Joint Surgery (American), 49, 807-835.

[9] Aronson, D.D. and Carlson, W.E. (1992) Slipped Capital Femoral Epiphysis: A Prospective Study of Fixation with a Single Screw. Journal of Bone and Joint Surgery (American), 74, 810-819.

[10] Boyer, D.W., Mickelson, M.R. and Ponseti, I.V. (1981) Slipped Capital Femoral Epiphysis: Long-Term Follow-Up Studies on One Hundred and Twenty-One Patients. Journal of Bone and Joint Surgery (American), 63, 85-95.

[11] Mulholland, R.C. and Gunn, D.R. (1972) Sliding Screw Plate Fixation of Intertrochanteric Femoral Fractures. Journal of Trauma-Injury Infection \& Critical Care, 12, 581-591. http://dx.doi.org/10.1097/00005373-197207000-00006

[12] Aronson, D.D. and Carlson, W.E. (1992) Slipped Capital Femoral Epiphysis: A Prospective Study of Fixation with a Single Screw. Journal of Bone and Joint Surgery (American), 74, 810-819.

[13] Fallath, S. (2004) Slipped Capital Femoral Epiphysis: An Analysis of Treatment Outcome According to Physeal Stability. Canadian Journal of Surgery, 47, 284-289.

[14] Tokmakova, K.P., Stanton, R.P. and Mason, D.E. (2003) Factors Influencing the Development of Osteonecrosis in Patients Treated for Slipped Capital Femoral Epiphysis. Journal of Bone and Joint Surgery (American Volume), 85, 798-801.

[15] Zaltz, I., Baca, G. and Clohisy, J.C. (2013) Unstable SCFE: Review of Treatment Modalities and Prevalence of Osteonecrosis. Clinical Orthopaedics and Related Research.

[16] Casey, B.H., Hamilton, H.W. and Bobechko, W.P. (1972) Reduction of Acutely Slipped Upper Femoral Epiphysis. Journal of Bone \& Joint Surgery (British), 54B, 607-614.

[17] Kalogrianitis, S., Tan, C.K., Kemp, G.J., Bass, A. and Bruce, C. (2007) Does Unstable Slipped Capital Femoral Epiphysis Require Urgent Stabilization. Journal of Pediatric Orthopaedics B, 16, 6-9. http://dx.doi.org/10.1097/01.bpb.0000236224.14325.f1

[18] Gordon, J.E., Abrahams, M.S., Dobbs, M.B., Luhmann, S.J. and Schoenecker, P.L. (2002) Early Reduction, Arthrotomy, and Cannulated Screw Fixation in Unstable Slipped Capital Femoral Epiphysis Treatment. Journal of Pediatric Orthopaedics, 22, 352-358. http://dx.doi.org/10.1097/01241398-200205000-00017

[19] Peterson, M.D., Weiner, D.S., Green, N.E. and Terry, C.L. (1997) Acute Slipped Capital Femoral Epiphysis: The Value and Safety of Urgent Manipulative Reduction. Journal of Pediatric Orthopaedics, 17, 648-654. http://dx.doi.org/10.1097/01241398-199709000-00013

[20] Tokmakova, K.P., Stanton, R.P. and Mason, D.E. (2003) Factors Influencing the Development of Osteonecrosis in Patients Treated for Slipped Capital Femoral Epiphysis. Journal of Bone and Joint Surgery (American), 85, 798-801.

[21] Loder, R.T. (2001) Unstable Slipped Capital Femoral Epiphysis. Journal of Pediatric Orthopaedics, 21, 694-699. http://dx.doi.org/10.1097/01241398-200109000-00027

[22] Herrera-Soto, J.A., Duffy, M.F., Birnbaum, M.A. and Have, V. (2008) Increased Intracapsular Pressures after Unstable Slipped Capital Femoral Epiphysis. Journal of Pediatric Orthopaedics, 28, 723-728. http://dx.doi.org/10.1097/BPO.0b013e318186bda3

[23] Sankar, W.N., McPartland, T.G., Millis, M.B. and Kim, Y.J. (2010) The Unstable Slipped Capital Femoral EpiphysisRisk Factors for Osteonecrosis. Journal of Pediatric Orthopaedics, 30, 544-548. http://dx.doi.org/10.1097/BPO.0b013e3181e4f372

[24] Tudiso, C., Caterini, R., Farsetti, P. and Potenza, V. (1999) Chondrolysis of the Hip Complicating Slipped Capital Femoral Epiphysis: Long-Term Follow-Up of Nine Patients. Journal of Pediatric Orthopaedics B, 8, 107-111.

[25] Rattey, T., Piehl, F. and Wright, J.G. (2002) Acute Slipped Capital Femoral Epiphysis: Review of Outcome and Rates of Avascular Necrosis. Journal of Bone and Joint Surgery (American Volume), 78, 398-402. 
Scientific Research Publishing (SCIRP) is one of the largest Open Access journal publishers. It is currently publishing more than 200 open access, online, peer-reviewed journals covering a wide range of academic disciplines. SCIRP serves the worldwide academic communities and contributes to the progress and application of science with its publication.

Other selected journals from SCIRP are listed as below. Submit your manuscript to us via either submit@scirp.org or Online Submission Portal.
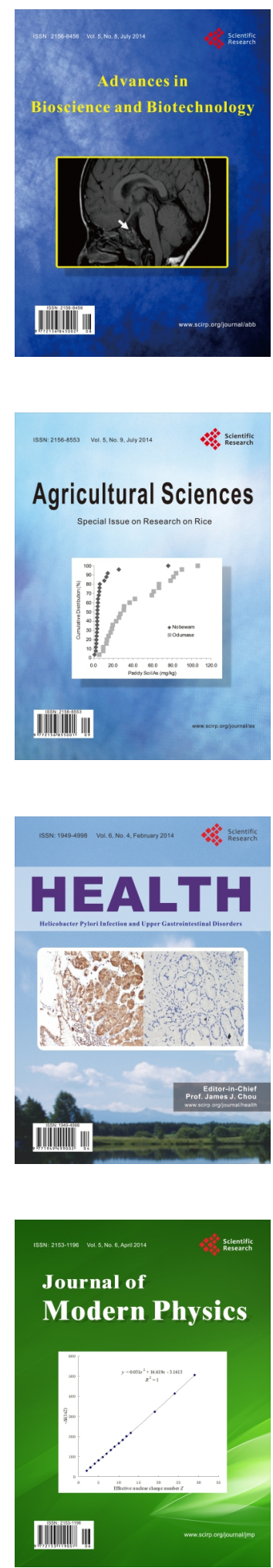
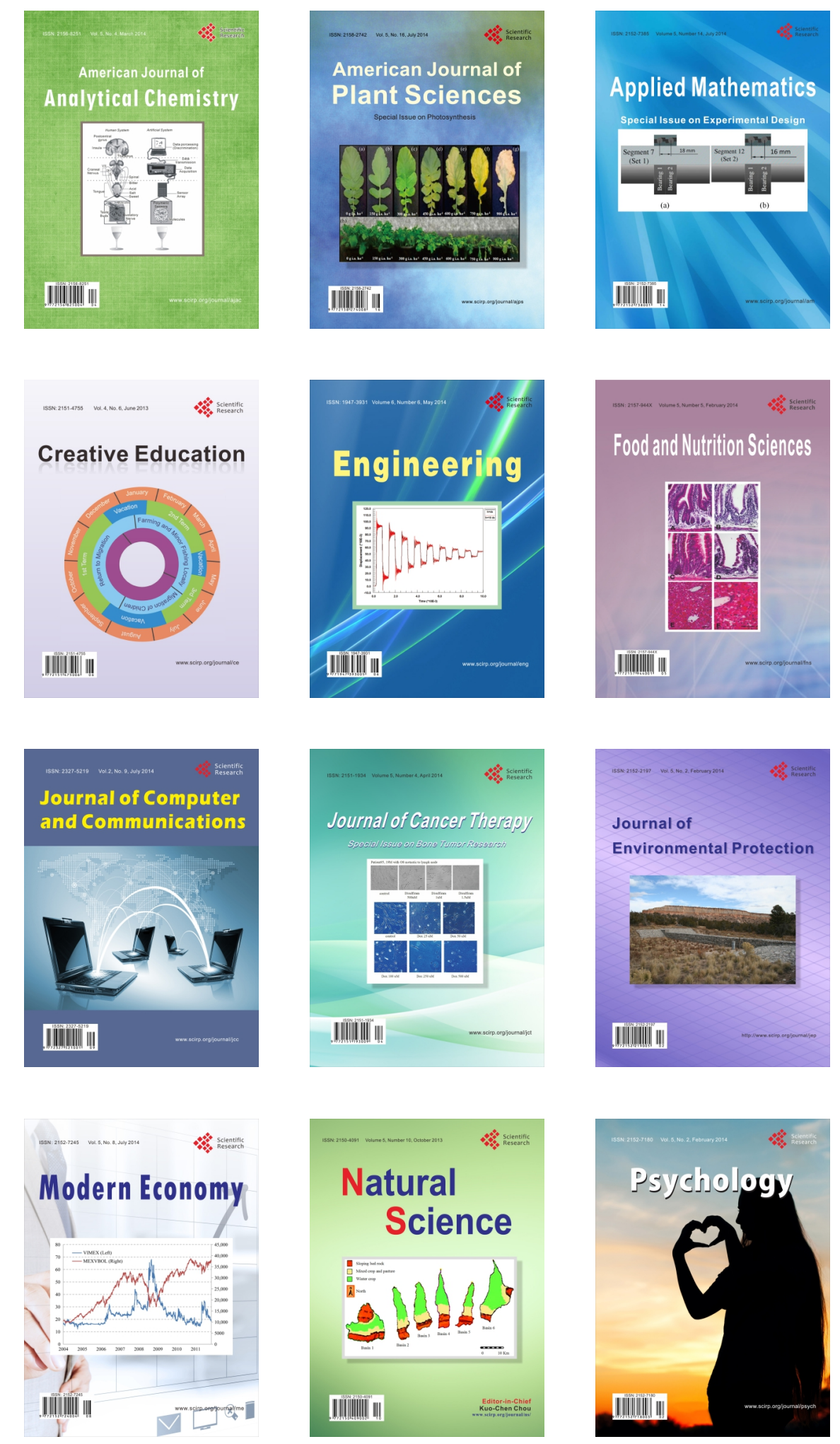